\title{
Effect of breathing techniques on blood pressure response to resistance exercise
}

\author{
Steven T. Linsenbardt MA, Tom R. Thomas PhD* and Richard W. Madsen PhDt \\ Hammons Heart Institute, Springfield, MO, USA; *Department of Health and Physical Education, University of \\ Missouri, Columbia, MO 65211, USA; +Department of Statistics, University of Missouri, Columbia, MO 65211, \\ USA
}

\begin{abstract}
Twenty novice male weight lifters performed resistance exercises using three different breathing techniques to determine the effects on blood pressure. Systolic and diastolic blood pressures were measured by an automated non-invasive method while subjects performed the single arm curl and double knee extension using the different breathing techniques. Performing the Valsalva manoeuvre (breath-holding) during either the single arm curl or double knee extension produced the highest blood pressure responses. Inhaling during the concentric phase of the exercise was associated with blood pressure elevations that were similar to the elevations observed with exhaling during the concentric phase. The heart rate response was slightly higher with inhalation. These results suggest that performing the Valsalva manoeuvre exaggerates the blood pressure response to resistance exercise. In addition, coupling inhalation with the concentric phase of the lift offers no cardiovascular advantage over coupling exhalation with the concentric phase of the lift.
\end{abstract}

Keywords: Weight training, blood pressure, total peripheral resistance, intrathoracic pressure

Several investigators have observed increased systolic (SBP) and diastolic blood pressure (DBP) in individuals performing heavy resistance exercise ${ }^{1-3}$. This $\mathrm{BP}$ response presumably is caused in part by the increased vascular total peripheral resistance in the contracting muscle ${ }^{3}$. In addition to this peripheral factor, the BP response may be augmented by increased intrathoracic pressure which occurs during forced exhalation against a closed airway (Valsalva manoeuvre) or by exhalation alone $e^{4,5}$. Exhalation or breath-holding may add to the increased afterload on the heart and thus increase BP. On the other hand inhalation, which decreases intrathoracic pressure and thus afterload ${ }^{4}$, may help attenuate BP elevation associated with resistance exercise.

The peripheral resistance in muscle apparently depends on the type of contraction. BP responses are much higher during concentric contractions than eccentric $^{2,3}$. Therefore, by combining the smaller

Address for correspondence: Tom R. Thomas, Department of Health and Physical Education, 38 Rothwell Gymnasium,

University of Missouri, Columbia, MO 65211, USA

(C) 1992 Butterworth-Heinemann Ltd

0306-3674/92/020097-04 afterload phase of breathing (inhalation) with the higher afterload phase of lifting (concentric), the BP response may be lessened.

The purpose of this study was to determine if breathing technique affected BP response during resistance exercise. If this $\mathrm{BP}$ effect can be dampened, resistance exercise may be safer for all populations but especially for those who are untrained, at high risk for cardiovascular disease, or have diagnosed cardiovascular disease.

\section{Methods}

Twenty men, aged 18-36 years, volunteered for this study. Their physical characteristics are given in Table 1 . During the 2 months before the study, each subject averaged 3 days per week of moderate resistance training in which all major muscle groups were used. Eleven of the 20 subjects participated in some form of weekly aerobic exercise. No subject was hypertensive $(>140 / 90 \mathrm{mmHg})$. Informed consent was obtained according to the policies of the Helsinki Declaration, and all procedures were approved by the University Institutional Review Board.

On the initial visit to the laboratory, each subject was screened for hypertension and had body composition estimated by skinfolds taken from the chest, abdomen, and thigh ${ }^{6}$. In addition, a 10 repetition maximum (RM) was established for the double knee extension on the Universal Gym and for the seated left-handed single arm curl using a dumbbell. Lifting speed and breathing patterns were practised.

Table 1. Physical characteristics of 20 male subjects

\begin{tabular}{lcc}
\hline & Mean & s.d. \\
\hline Age (years) & 23.4 & 4.6 \\
Height $(\mathrm{cm})$ & 177.6 & 8.0 \\
Weight $(\mathrm{kg})$ & 74.6 & 8.3 \\
Body fat $(\%)$ & 8.5 & 3.4 \\
Resting SBP & 121 & 9 \\
Resting DBP & 76 & 8 \\
Double knee extension 10 RM (kg) & 15.3 & 3.4 \\
Single arm curl 10 RM (kg) & 9.1 & 1.3 \\
\hline
\end{tabular}

SBP, DBP, systolic and diastolic blood pressures; RM, repetition maximum 
For the second visit, each subject abstained from exercise for $24 \mathrm{~h}$ and performed, in a random order, three sets of $10 \mathrm{RM}$ on each resistance exercise: (1) inhalation coupled with the concentric phase and exhalation coupled with the eccentric phase, (2) exhalation coupled with the concentric phase and inhalation coupled with the eccentric phase, (3) Valsalva in which the breath was held during two-thirds of the concentric phase followed by exhalation during the remainder of the repetition.

Three-minute rests were given between sets, and the six sets were alternated between the upper and lower body, so that 7-8 min rests were given between any two sets using the same muscle group. For both exercises, a metronome was used to maintain a constant lifting velocity at $30 \%$. The weight was moved through the full range of motion. The subject's position was standardized, and the torso was stabilized to ensure that only the targetted muscle group was significantly active.

During each repetition, BP was measured in the right arm using a physiograph (E \& M Instrument Co., Houston, Texas, USA) in which an internal pump automatically inflated the arm cuff. The cuff was deflated manually. A sensor in the cuff detected each Korotkoff sound and transmitted the signal to the recorder. Before each test, the physiograph and recorder were calibrated with a standard mercury column. It was desirable to measure each BP at multiple times during each set; therefore, the cuff pressure was maintained at the systolic level during the concentric phases of repetitions 6, 7 and 8. The cuff was then deflated, and DBP was measured during the concentric phases of repetitions 9 and 10 . A mean value was calculated for both SBP and DBP. Heart rate was assessed from the BP recordings during repetitions 6,7 and 8 . All values were expressed as mean(s.d.). Analysis of variance with repeated measures was used to compare differences in BP and heart rate among breathing techniques. Significant $F$ ratios $(P<0.05)$ were followed by Tukey post hoc analyses.

\section{Results}

Reliability of the procedure for SBP was $r=0.90$ on 24 pairs of repeated lifting trials. For DBP the reliability was $r=0.95$ on 11 pairs of repeated trials.

Figure 1 illustrates the average SBP and DBP during the double knee extension. Significantly higher SBP and DBP occurred using the Valsalva $(166 / 108 \mathrm{mmHg})$ breathing technique than inhalation (151/99). Although both BPs were higher during exhalation $(159 / 102)$ than inhalation, this difference did not achieve statistical significance.

Likewise, on the single arm curl, SBP and DBP were higher using the Valsalva (166/112) than the inhalation technique (148/101) (Figure 2). Exhalation $(156 / 105)$ versus inhalation and exhalation versus Valsalva techniques were not statistically different in either $\mathrm{BP}$ response. In addition, the $\mathrm{BP}$ responses during the double knee extension and the single arm curl for any given breathing technique were very similar.

Mean heart rate during the double knee extension was similar regardless of breathing technique. However, mean(s.d.) heart rate during the single arm curl was significantly higher during the inhalation (101(11)b.p.m.) than the exhalation technique (94(12) b.p.m.), but not the Valsalva manoeuvre (97(9) b.p.m.) technique (Figure 3).

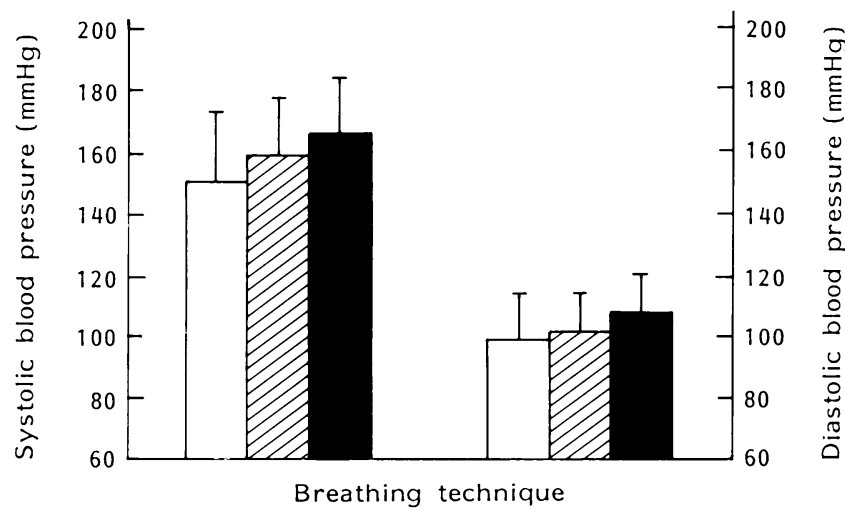

Figure 1. Systolic and diastolic blood pressures during the double knee extension. Inhale $(\square)$, exhale (G), and Valsalva (ם) breathing were each coupled with the concentric phase of the lift. Inhale and Valsalva values are significantly different for both pressures. Height of bar $=$ mean, length of line $=$ s.d.

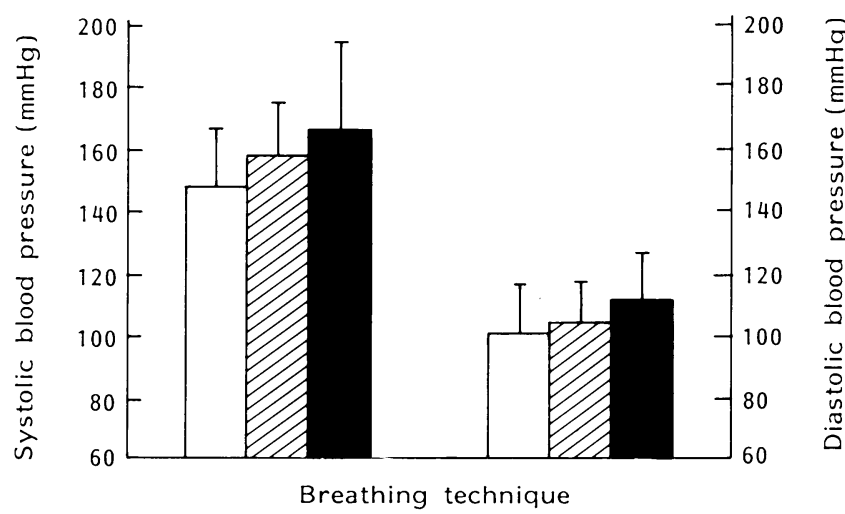

Figure 2. Systolic and diastolic blood pressures during the single arm curl with inhale ( $\square$ ), exhale (目), and Valsalva (ם) breathing. Inhale and Valsalva values are significantly different for both pressures. Height of bar = mean, length of line $=$ s.d.

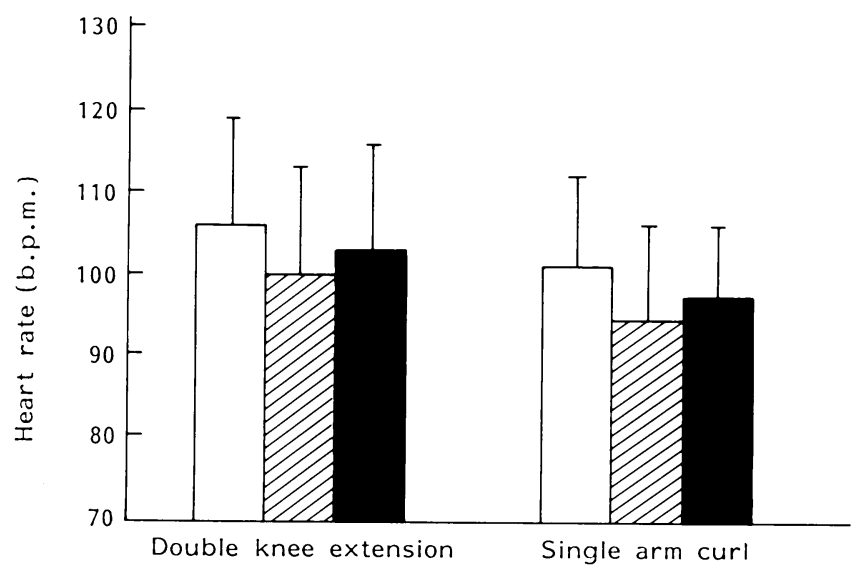

Figure 3. Heart rates during the double knee extension and single arm curl with inhale ( $\square$ ), exhale (目), and Valsalva (घ) breathing. No significant differences existed for the leg exercise. Inhale and exhale values are significantly different for the arm exercise. Height of bar = mean, length of line $=$ s.d. 


\section{Discussion}

The mean blood pressure responses during resistance exercise observed in the present study are slightly lower than those reported by others using intraarterial pressure methods. For example, a mean of $160 / 120$ was reported by Fleck and Dean ${ }^{1}$ for body builders using the single arm curl at $80 \% 1 \mathrm{RM}$ to fatigue. In addition, Freedson $e t$ al. ${ }^{7}$ reported a mean $B P$ of $169 / 108$ in men performing 10 repetitions on a bench press at $50 \% 1 \mathrm{RM}$. The intra-arterial pressure transducer used to measure BP in these studies has been shown to produce higher SBP and DBP values during exercise than auscultation techniques, ${ }^{8,9}$. However, only Wiecek et al. ${ }^{10}$ have systematically compared direct and indirect blood pressure measurements during resistance exercise. In that study, auscultation underestimated SBP by a consistent $20-25 \mathrm{mmHg}$ during lifting at $40 \%$ or $60 \%$ of maximal load. DBP during lifting did not differ between direct and indirect methods, and recovery pressure comparisons were more variable. Thus, the SBP measured during exercise in the present study may have underestimated true intra-arterial pressure. However, the comparison among breathing trials should be valid since Wiecek et al. ${ }^{10}$ determined that any underestimation during lifting is consistent across subjects and exercises.

The SBP and DPB observed in the present study are similar to those obtained using auscultation methods by Misner et al. ${ }^{11}$ during sustained isometric leg extension and by Miles et al. ${ }^{3}$ during 12RM leg extensions in strength trained men. Only Mac-

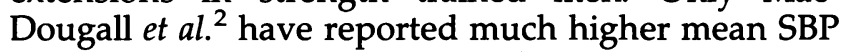
and DBP values during arm curls and leg presses in experienced body builders who were allowed to perform a Valsalva manoeuvre. In the present study, the most trained and heavily muscled subject had SBP values considerably higher than the other subjects and similar to those observed by MacDougall et al. ${ }^{2}$.

Although the magnitude of the response differs among studies using different populations, research is very consistent regarding the direction of the SBP and DBP response to resistance exercise. As suggested by Miles et $a l .{ }^{3}$, the increased pressure is most likely caused by the partial occlusion of blood flow induced by the contracting muscles. Our data and those of $\mathrm{O}^{\prime}$ Connor et al. ${ }^{12}$ suggest that this increased total peripheral resistance can be exaggerated by increased intrathoracic pressure induced by the Valsalva manoeuvre.

It is possible that the BP response for each treatment in the present study was affected by the order of the lifts, i.e. the third sets may have been more difficult than the first and induced larger pressure elevations. Although all sets were performed during the same session, the order was randomized for each subject. In addition, at least $7 \mathrm{~min}$ separated any two sets involving the same muscle group. In all cases, the subject was able to complete the $10 \mathrm{RM}$ on the third set. In seven subjects in which recovery BP was monitored, the mean value after recovery $(3 \mathrm{~min})$ from the third set was 130/72 and after recovery from the sixth set in the same session was $133 / 79$. Taken together, the randomization of trials and rapid BP recovery suggest that the order of treatment did not affect the results of this study.

In the present study, the SBP elevations were similar to those observed during aerobic exercise although DBP in this study was consistently higher during resistance exercise than during aerobic exercise $^{13}$. The pressure values reported by MacDougall et $a l^{2}$ indicate that for some resistance exercises the SBP and DBP response can be considerably higher than those usually observed in moderate aerobic exercise. The danger of these acute increases in BP is unknown. They do not appear to cause increases in resting $\mathrm{BP}^{1,13,14}$, but may lead to myocardial hypertrophy of unknown benefit ${ }^{15,16}$ or even to severe headaches ${ }^{17}$.

The results of Fox et al. ${ }^{4}$ suggest that the BP elevation induced by performing the Valsalva manoeuvre during exercise may be underestimated. In their study, the largest rise in SBP was observed several seconds after the manoeuvre (stage IV or overshoot). Immediate post-exercise BP was not measured in the present study. However, this delayed response may help explain the gradual increase in SBP during successive repetitions as observed in the present study and by MacDougall $e t$ $a .^{2}$.

A number of resistance training experts believe that performing the Valsalva manoeuvre actually benefits the lifter by stabilizing the spine and improving performance ${ }^{2,18}$. These potential benefits must be weighed against the potential dangers of the exaggerated pressure response. A prudent recommendation would be to avoid this breath-holding technique when lifting or when performing other heavy resistance activities, such as snow shovelling, wood chopping and pushing. SBP appears to climb during successive repetitions of the set and can reach very high values on the final repetition in certain individuals. Such maximal efforts perhaps should be reserved for young competitive athletes and others who require maximal strength gains for sport performance.

Our results indicate that the BP response during resistance exercise may be attenuated by inhaling during the concentric phase of the lift. Many individuals are more comfortable with the opposite breathing technique, i.e. coupling the concentric phase with exhalation. In practice, this alternate method seems appropriate since we observed only small differences in the pressure response between the inhalation and exhalation techniques. In fact, the exhalation technique produced lower heart rates (HR) than inhalation and thus the double products (SBP $\times$ HR) for inhalation and exhalation were comparable.

\section{Conclusion}

The results of this study suggest that the Valsalva manoeuvre exaggerates the blood pressure response to resistance exercise. Coupling inhalation or exhalation with the concentric phase of the lift produces similar cardiovascular responses. More research examining breathing techniques during resistance 
exercise in other populations such as competitive athletes appears warranted.

\section{Acknowledgement}

The authors thank Dr John A. Roberts for his helpful suggestions on the design of the study.

\section{References}

1 Fleck SJ, Dean LS. Resistance training experience and the pressor response during resistance exercise. J Appl Physiol 1987; 63: 116-20.

2 MacDougall JD, Tuxen D et al. Arterial blood pressure response to heavy resistance training. J Appl Physiol 1985; 58: 785-90.

3 Miles DS, Owens JJ, Golden JC, Gotschall RW. Central and peripheral hemodynamics during maximal leg extension exercise. Eur J Appl Physiol 1987; 56: 12-17.

4 Fox IJ, Crowley WP, Grace JB, Wood EH. Effects of the Valsalva maneuver on blood flow in the thoracic aorta in men. J Appl Physiol 1966; 21: 1553-60.

5 Milnor WR. Pulmonary circulation. In: Mountcastle VB, ed. Medical Physiology. St Louis, USA: CV Mosby, 1974: 1015-16.

6 Jackson AS, Pollock ML. Generalized equations for predicting body density of men. Br I Nutr 1978; 40: 497-504.

7 Freedson $\mathrm{P}$, Chang B, Katch $\mathrm{F}$ et al. Intra-arterial blood pressure during free weight and hydraulic resistance exercise. Med Sci Sports Exerc 1984; 16: 131 (Abstract).
8 Robinson TE, Sue DY, Huszczuk A et al. Intra-arterial and cuff blood pressure responses during incremental cycle ergometry. Med Sci Sports Exerc 1988; 20: 142-9.

9 Nagle FJ, Naughton J, Balke B. Comparison of direct and indirect blood pressure with pressure flow dynamics during exercise. J Appl Physiol 1966; 21: 317-20.

10 Wiecek EM, McCartney N, McKelvie RS, MacDougall D. Indirect vs direct measures of arterial pressure during weight lifting in cardiac patients. Med Sci Sports Exerc 1990; 16: 131 (Abstract).

11 Misner JE, Gorny SB, Massey BH et al. Cardiovascular response to sustained maximal voluntary static muscle contraction. Med Sci Sports Exerc 1990; 22: 194-9 (Abstract).

12 O'Connor P, Sforzo GA, Frye PA. The effects of breathing techniques upon blood pressure during isometric exercise. Med Sci Sports Exerc 1987; 19: S94 (Abstract).

13 Astrand PO, Ekblom B, Messin R et al. Intra arterial blood pressure during exercise with different muscle groups. J Appl Physiol 1965; 20: 253-9.

14 Hagberg JM, Ehsani AA, Goldring D et al. Effect of weight training on blood pressure and hemodynamics in hypertensive adolescents. J Pediatr 1984; 104: 147-51.

15 Harris KA, Holly RG. Physiological response to circuit weight training in borderline hypertensive subjects. Med Sci Sports Exerc 1987; 19: 246-52.

16 Kraemer WJ, Deschenes MR, Fleck SJ. Physiological adaptations to resistance exercise: implications for athletic conditioning. Sports Med 1988; 6: 246-56.

17 Carswell H. Headaches: a weighty problem for lifters? Physician Sports Med 1984; 12: 23.

18 Austin D, Roll F, Kreis EJ et al. Breathing during weight training. Natl Strength Condition Assoc J 1987; 9: 17-25. 